\title{
ACOPLAMENTO CROMATOGRAFIA GASOSA - ESPECTROMETRIA DE ABSORÇÃO ATÔMICA EM ESTUDOS
} DE ESPECIAÇÃO: UMA REVISÃO

\section{Reinaldo Calixto de Campos e Patricia Grinberg}

Departamento de Química, Pontifícia Universidade Católica do Rio do Janeiro, Rua Marquês de São Vicente, 225, 22453-900 Rio de Janeiro - RJ

Recebido em 13/3/00; aceito em 7/8/00

\begin{abstract}
GAS CHROMATOGRAPHY COUPLED WITH ATOMIC ABSORPTION SPECTROMETRY. This review presents an updated overview of the trace element speciation by gas chromatography coupled with atomic absorption spectrometry.
\end{abstract}

Keywords: speciation; gas cromatography; atomic absorption spectrometry.

\section{INTRODUÇÃO}

O termo especiação foi definido por Ure ${ }^{1}$ como um processo ativo de identificação e quantificação de diferentes espécies, formas ou fases em que um elemento ocorre em uma determinada amostra. A importância da especiação para a ciência ambiental, biologia e medicina reflete-se na crescente quantidade de artigos e livros publicados nos últimos anos, de congressos devotados ao tema e no constante progresso alcançado. A principal razão é que a toxicidade, bio-disponibilidade, o transporte e propriedades físico-químicas de um elemento podem diferir grandemente, dependendo de sua forma química ${ }^{2,3}$. Logo, informar o conteúdo total de um elemento não é suficiente na avaliação de seu potencial de ação. Por exemplo, o Sn inorgânico apresenta menor toxicidade do que os seus compostos alquilderivados, sendo que a toxicidade aumenta com o aumento do número de grupos alquila ligados ao átomo de $\mathrm{Sn}^{4}$. Entretanto, para o As, os compostos inorgânicos são mais tóxicos do que os respectivos compostos orgânicos, o mesmo ocorrendo com os compostos de $\mathrm{Sb}$. Já em relação à influência do número de oxidação, sabe-se, por exemplo, que o As(III) é mais tóxico que o $\mathrm{As}(\mathrm{V})$ e que o $\mathrm{Sb}(\mathrm{III})$ se mostra dez vezes mais tóxico do que $\mathrm{Sb}(\mathrm{V})^{5,6}$. Em relação aos compostos de mercúrio, tanto as espécies inorgânicas como as orgânicas são tóxicas, sendo seus alquil derivados os mais tóxicos, em especial o metil-mercúrio, de todas as formas, a mais agressiva ${ }^{7}$.

Logo, o desenvolvimento de ferramentas analíticas precisas e seletivas para a determinação destas diferentes espécies é de extrema importância para uma estimativa realista dos riscos toxicológicos ou do comportamento ambiental de um dado elemento ${ }^{8}$.

A determinação individual das diferentes espécies de um elemento, presente a nível de traços, é dependente dos seguintes requisitos ${ }^{9}$ :

- Os compostos de interesse devem ter suas integridades preservadas durante a amostragem, armazenamento e pré-tratamento da amostra. Deve-se evitar qualquer ação que resulte em uma mudança do equilíbrio químico, destruição ou transformação das diferentes formas existentes na amostra. Este requisito é considerado o principal problema da especiação;

- As análises devem ser específicas e não sujeitas a interferências de outros elementos ou compostos presentes na amostra;

- No caso de processos de separação, estes devem ser, além de eficientes, tais que não impliquem em excessiva diluição das espécies;

- O método de detecção deve ser suficientemente sensível, de modo a permitir determinações a nível de traços e ultra-traços.
Assim, os métodos analíticos aplicados à determinação seletiva das espécies elementares podem ser classificados como:

- métodos químicos, baseados em técnicas de separação por extração, volatilização, co-precipitação ou por redução seletiva;

- métodos cinéticos;

- métodos baseados em técnicas cromatográficas.

Os métodos baseados em separações não cromatográficas, assim como os cinéticos, apresentam boa precisão e têm a vantagem de ter baixo custo operacional, pois necessitam de instrumentação bastante simples; entretanto, podem ser bastante laboriosos e demorados.

Já os métodos baseados em técnicas cromatográficas, apesar de necessitarem de instrumentação mais sofisticada, são mais eficientes na separação das diferentes espécies, sendo, portanto, os mais empregados, especialmente na especiação de compostos organometálicos em amostras clínicas ou ambientais. A maior parte dos sistemas visa o acoplamento direto da cromatografia, em linha, a técnicas de detecção diversas, como AAS, ICP-MS ${ }^{10-21}$, ICP-AES ${ }^{10,22-29}$, AFS30, MIP ${ }^{10,21,31-46}$ etc. Ou seja, os diferentes equipamentos relativos a essas técnicas analíticas tornam-se os detectores, postados à saída da coluna de separação.

Nestes acoplamentos, a cromatografia líquida, principalmente HPLC, tem sido uma técnica amplamente utilizada ${ }^{47}$, tanto devido a seu alto poder de separação, como pelo fato de poder lidar com analitos voláteis ou não. Entretanto, a instrumentação pode apresentar-se cara e o tempo de análise excessivamente longo. Concomitantemente, a sua interface com técnicas de espectrometria atômica implica, na maioria dos casos, na nebulização pneumática do efluente, com as perdas que este modo de introdução de amostra acarreta, ou no uso de sistemas especiais, não disponíveis comercialmente. Para alguns elementos, a nebulização pode também ser contornada por reações de derivatização a formas voláteis, após a coluna, o que implica, entretanto, no aumento da complexidade do sistema.

Frente à HPLC, além do mais baixo custo, a cromatografia gasosa tem a vantagem de transferir ao detector o analito já na forma gasosa, evitando assim os problemas de perdas inerentes à nebulização da amostra, aumentando, consequentemente, a sensibilidade do método. No entanto, sua utilização é limitada a compostos voláteis, ao passo que grande parte dos problemas de especiação envolve espécies não voláteis. Contudo, esta limitação também pode ser contornada pela derivatização das espécies de interesse a compostos voláteis.

Entre todas as técnicas analíticas utilizadas para detecção, grande atenção tem sido dada ao ICP-MS, que é considerado o detector mais sensível para a especiação e que terá uma 
importância crescente no futuro, principalmente onde é necessária a melhor sensibilidade e/ou a análise simultânea de diversos elementos. Entretanto, seu alto custo e conseqüente indisponibilidade, faz com que a AAS seja considerada a técnica de maior potencial de uso em análises de rotina, onde o custo se torna um fator determinante para a escolha do método ${ }^{47}$.

A AAS, além de ser uma técnica relativamente barata, tanto na sua implantação quanto nos gastos de operação, é de fácil operação, estando disponível na maioria dos laboratórios, e o acoplamento de técnicas de derivatização, como geração de hidretos ou etilação, não traz qualquer dificuldade maior. Dependendo do elemento e forma a ser determinada, a interface GC-AAS pode ser bastante simples, usufruindo da AAS sua sensibilidade, robustez e especificidade.

Para a melhor utilização do acoplamento GC-AAS ${ }^{47-49}$, as seguintes condições devem ser observadas ${ }^{50}$ :

1. Todas espécies injetadas na coluna cromatográfica devem ser quantitativamente eluídas, sem que ocorra decomposição ou transformação na coluna;

2. O fluxo do gás que entra na célula de detecção deve ser constante durante toda a corrida cromatográfica;

3. Uma vez estando na célula de detecção, todos os analitos devem ser distribuídos uniformemente na seção do atomizador e completa e instantaneamente atomizados.

No sistema GC-AAS, o procedimento de leitura pode ser caracterizado por, pelo menos, 3 etapas, conforme Figura 1:

- Introdução da amostra na coluna, precedida ou não, da derivatização

- Eluição e separação dos compostos presentes na amostra e introdução destes no AAS, através da interface;

- Atomização de cada um destes compostos e detecção

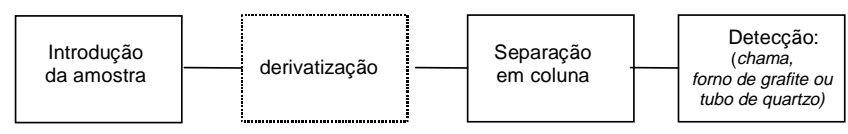

Figura 1. Esquema do acoplamento CG-AAS.

\section{INTRODUÇÃO DA AMOSTRA}

$\mathrm{Na}$ cromatografia gasosa, podem-se utilizar amostras gasosas ou líquidas, estas últimas desde que sejam totalmente volatilizadas dentro da coluna. Analitos no estado gasoso, a partir de amostras líquidas, podem ser gerados termicamente ou através de técnicas de derivatização (geração de hidretos, etilação etc). A derivatização, além de permitir a determinação de espécies de outro modo não voláteis, leva à separação do analito da matriz, o que irá diminuir ou até mesmo eliminar as interferências no processo de separação e atomização posteriores, pois apenas um pequeno número de concomitantes acompanha as espécies assim volatilizadas.

Dentre as técnicas de derivatização, a geração de hidretos é a mais utilizada ${ }^{51}$; neste processo, diversos elementos, como As, $\mathrm{Bi}, \mathrm{Se}, \mathrm{Sb}, \mathrm{Sn}, \mathrm{Te}, \mathrm{Ge}, \mathrm{In}, \mathrm{Pb}$ e Cd, e suas respectivas espécies químicas podem ser levadas a seus respectivos hidretos gasosos. A redução é feita a partir da geração de hidrogênio nascente, pela adição de $\mathrm{NaBH}_{4}$ a uma solução acidificada.

Uma vez gerado, o hidreto pode ser transferido, com a ajuda de um gás de arraste, diretamente à coluna. A reação de redução e a separação gás/líquido do hidreto gerado é facilmente adaptável para sistemas em linha, havendo várias versões disponíveis no mercado.

O mercúrio é um caso especial, pois forma $\mathrm{Hg}^{\circ}$ pela redução com o $\mathrm{NaBH}_{4}$, tanto a partir do íon $\mathrm{Hg}^{2+}$, como a partir de organoderivados, inclusive o metilmercúrio. Assim, sua especiação não se faz possível pelo $\mathrm{NaBH}_{4}$. Outra dificuldade com o uso do $\mathrm{NaBH}_{4}$ é causada pelo fato de alguns compostos organoderivados dos elementos supracitados não serem por ele redutíveis ou apresentarem cinética de reação extremamente lenta ${ }^{52}$.

Em substituição à derivatização pelo $\mathrm{NaBH}_{4}$, tem sido cada vez mais utilizada a alquilação. Esta é feita a partir de reagentes de Grignard ${ }^{53-56}$ ou de alquil derivados do borohidreto de sódio, como o tetraetilborato de sódio ${ }^{7,54,57-60}$ e o tetrapropilborato de sódio ${ }^{40}$, que geram alquil derivados dos elementos e espécies em questão. O processo de alquilação e liberação da solução dos alquilderivados formados pode ser muito mais lento do que na geração de hidretos, necessitando de até 15 minutos, o que dificulta a utilização de sistemas em linha. A alquilação pode levar, ainda, a uma melhor diferenciação das espécies ${ }^{40}$ ou ser indicada no caso de instabilidade das moléculas dos hidretos, como é o caso da determinação de $\mathrm{Pb}^{57,61-62}$. A eficiência da derivatização irá depender, entretanto, da matriz envolvida e do composto de interesse. Por exemplo, Cai e colaboradores $^{59}$ realizaram um estudo onde foram comparadas a utilização de borohidreto de sódio e tetraetilborato de sódio para a derivatização dos compostos de estanho (monobutil, dibutil e tributilestanho), em sedimentos. Verificaram que os compostos monobutil e dibutilestanho podem ser quantitativamente determinados pela utilização do método da geração de hidretos $\left(\mathrm{NaBH}_{4}\right.$ como redutor); porém, a espécie tributil sofria algumas interferências críticas pelo extrato do sedimento. Com a utilização da etilação ( $\mathrm{NaBEt}_{4}$ como redutor), pode-se alcançar uma determinação quantitativa das espécies dibutil e tributilestanho, embora tenha sido observada uma baixa recuperação da espécie monobutílica.

Uma outra estratégia para a introdução de amostras em sistemas CG-AAS pode ser a utilização da captura criogênica ${ }^{63}$, indicada para compostos de baixo ponto de ebulição. Neste caso, um tubo de vidro silanizado, em U, resfriado por um banho de nitrogênio líquido, retém, por condensação, os compostos voláteis gerados. Após a coleta de todos os compostos, o banho de nitrogênio líquido é retirado e o tubo em $U$ é aquecido, eletricamente ou por imersão em banho de água quente, produzindo uma volatilização diferencial dos compostos, de acordo com seus pontos de ebulição. Estes são carreados para a coluna pelo gás de arraste, que pode ser argônio ou nitrogênio. A captura criogênica é uma técnica útil para pré-concentrar as espécies de interesse, respondendo ao longo tempo da reações de alquilação, aumentando a sensibilidade. O sistema deve ser mantido seco, pois um acúmulo de água e gelo dentro do tubo em U poderá bloqueá-lo. Para isso, utilizam-se ou um banho de 2-propanol e gelo seco ou $\mathrm{CaCl}_{2}$ como agente de secagem; porém, deve-se ter cuidado na utilização deste último, pois poderá haver a perda dos analitos devido a sua adsorção no $\mathrm{CaCl}_{2}$.

\section{COLUNAS}

Pode-se verificar, na literatura referente ao acoplamento CG-AAS, que não são apresentados estudos detalhados quanto à escolha da coluna. Tanto colunas empacotadas ${ }^{64-67}$ (geralmente preenchidas com Chromosorb W revestidas com 3-10\% OV-100 ou Carbowax), quanto colunas capilares ${ }^{68-73}$, tem sido utilizadas. Porém, deve-se ter sempre em mente que uma escolha errada da fase estacionária poderá inviabilizar a especiação. No caso do uso da captura criogênica, verificou-se que os tempos de retenção dos vários compostos podem ser linearmente relacionados com seus pesos moleculares, e que nem a composição nem a granulometria da fase estacionária afetaria a separação ${ }^{18}$. Isto leva alguns autores a considerar este mais como um processo de destilação fracionada do que uma separação cromatográfica ${ }^{74}$.

\section{INTERFACES}

Uma vez separadas as espécies, estas devem ser transferidas diretamente da coluna cromatográfica ao detector, a fim de 
minimizar perdas do analito, alargamento dos picos e formação de cauda, havendo-se que evitar, ainda, reações que possam interferir na detecção. Como interface GC-AAS utilizamse tubos de aço inox ${ }^{75}$, alumínio ${ }^{76}$, pirex ${ }^{77}$, teflon ${ }^{78}$ ou tungstênio $^{79}$. É muito importante que estas conecções sejam devidamente aquecidas, a fim de que não ocorra condensação ou acúmulo de componentes voláteis ao longo do tubo, o que poderá levar ao empobrecimento da sensibilidade, devido a perdas do analito nas paredes do tubo, e a efeitos de memória. Geralmente, é mantida uma temperatura semelhante à da utilizada na coluna ${ }^{80}$. No caso específico do $\mathrm{Hg}$, para que a detecção possa ser feita pela técnica do vapor frio, o aquecimento da interface deve ser de tal ordem que permita a destruição dos compostos organomercuriais, de forma que alcancem o detector já na forma atômica ${ }^{81}$.

\section{DETECÇÃO}

Para a detecção por AAS, é necessária a produção de átomos livres do analito. Isto é conseguido com a utilização de células de atomização como a chama ar-acetileno, a chama óxido nitroso-acetileno, tubos de quartzo aquecidos (eletricamente ou por chama), pelo sistema "flame-in-tube" e em fornos de grafite.

\section{Chama}

Kolb e colaboradores ${ }^{82}$, em 1966, foram os primeiros a demonstrar a capacidade da AAS como detector para cromatografia gasosa. Neste trabalho, o efluente da coluna era introduzido no nebulizador da chama ar-acetileno. Este método de introdução é bastante insatisfatório, pois pode ocorrer a condensação de espécies voláteis na zona não aquecida do nebulizador, havendo, também, diluição da amostra na câmara de mistura ${ }^{83-84}$. Apesar destas desvantagens, este mesmo procedimento foi ainda utilizado por outros grupos ${ }^{85-86}$. Em uma modificação deste procedimento, Coker ${ }^{87}$ fez com que os efluentes da coluna fossem introduzidos diretamente no queimador, obtendo um limite de detecção de cerca de $20 \mathrm{ng}$, para o chumbo. Apesar desses esforços, pode-se afirmar que, de uma maneira geral, a utilização da chama, seja ela aracetileno ou óxido nitroso- acetileno, não se adequa aos problemas de especiação, devido à baixa sensibilidade proporcionada por esse atomizador.

\section{Fornos de Grafite}

Nas análises por GF-AAS, o programa de aquecimento possui de 3 a 4 etapas básicas, de modo a, seqüencialmente, permitir a evaporação do solvente em temperaturas relativamente baixas, seguido do aumento da temperatura, a fim de possibilitar a pirólise da amostra, alcançando-se, então, a atomização do analito em uma temperatura maior ainda. Porém, quando o forno de grafite é acoplado à cromatografia gasosa, a temperatura de atomização deve ser mantida constante, até que toda a amostra seja eluída da coluna, para evitar que os compostos organometálicos possam escapar da célula de atomização sem serem detectados. Segar ${ }^{79}$, por exemplo, utilizando um tubo de tungstênio dirigiu os efluentes da coluna cromatográfica para um forno de grafite mantido na temperatura de atomização, através do orifício de admissão de amostra, que foi previamente aumentado. Obteve um limite de detecção de aproximadamente $10 \mathrm{ng}$ para o $\mathrm{Pb}$. Em outro trabalho Radziuk e colaboradores ${ }^{76}$ utilizaram um tubo de tântalo como conector entre a coluna e o forno de grafite, obtendo um limite de detecção da ordem de 0,04 ng, para as diferentes espécies de $\mathrm{Pb}$.

$\mathrm{Um}$ atomizador de carbono, em formato $\mathrm{T}$, foi desenvolvido por Robinson e colaboradores ${ }^{88}$. Os efluentes da coluna eram transferidos a este atomizador por um tubo aquecido, de aço inoxidável. O limite de detecção para $\mathrm{Pb}$ tetrametila foi também de 0,04 ng. Já Robinson e colaboradores ${ }^{77}$ utilizaram como célula de atomização uma câmara de carbono. Os efluentes eram levados até esta câmara por um capilar de pyrex, aquecido a $110^{\circ} \mathrm{C}$; o limite de detecção para $\mathrm{Pb}$ tetrametila foi de $0,04 \mathrm{ng}$

Um outro enfoque foi adotado por Bye e colaboradores ${ }^{89}$, que conectaram um tubo de alumina à coluna cromatográfica, aquecida a $130^{\circ} \mathrm{C}$, em posição tangencial em relação a uma das extremidades do forno de grafite. Desta forma, foram obtidos limites de detecção de 0,12 ng para $\mathrm{Pb}$ tetrametila e $1,1 \mathrm{ng}$ para $\mathrm{Pb}$ tetraetila. Outra possibilidade foi explorada por De Jonghe e colaboradores ${ }^{90}$, que introduziram o efluente da coluna através das entradas dos gases de purga, forçando-o, assim, a percorrer todo o caminho ótico do forno pré-aquecido, a partir de suas duas extremidades.

Conforme pode-se observar, a utilização do forno de grafite em acoplamento a técnicas cromatográficas requer a modificação dos equipamentos normalmente empregados, pois estes não são projetados para a operação contínua, o que leva a maior deterioração do fornos e, consequentemente, a um maior custo de análise. Entretanto, os limites de detecção alcançados, da ordem do $\mu \mathrm{g} \mathrm{L}^{-1}$, são bem melhores do que aqueles obtidos com a chama.

\section{Tubos de Quartzo}

Um outro tipo de célula de atomização são os tubos de quartzo. Eles possuem um formato $\mathrm{T}$ e ficam alinhados no caminho ótico do espectrômetro, sendo que as amostras alcançam o volume de atomização pela sua haste central.

Chau e colaboradores ${ }^{91}$ utilizaram como célula de atomização um tubo de quartzo, de $7 \mathrm{~mm}$ de diâmetro interno e $6 \mathrm{~mm}$ de comprimento, aquecido eletricamente e com as extremidades abertas, para a determinação de compostos de chumbo tetra-alquila. Os gases efluentes da coluna cromatográfica eram levados diretamente à célula de atomização, misturados com $\mathrm{H}_{2}$, a fim de se melhorar a atomização. Os autores obtiveram um limite de detecção, para os diversos compostos de $\mathrm{Pb}$, de cerca de $0,1 \mathrm{ng}$, constatando um aumento de sensibilidade de 2 ordens de grandeza, quando comparando com a adição direta, na chama, dos efluentes da coluna cromatográfica. Em um outro estudo" ${ }^{92,}$ um tubo metálico ("liner") foi colocado dentro do tubo $\mathrm{T}$, para prevenir a adsorção dos analitos na superfície do quartzo.

Os tubos de quartzo são as células mais amplamente utilizadas no acoplamento GC-AAS, e são caracterizados por levarem a alta sensibilidade, serem de fácil operação e de baixo custo. Deve-se evitar, porém, a possibilidade de formação, no seu interior, de misturas de gases que possam entrar em combustão, o que interfere no tempo de residência dos átomos do analito no caminho ótico. Além disso, tubos de quartzo possuem uma temperatura limitada de uso, o que reduz o número de elementos que podem ser determinados, em comparação com os fornos de grafite. Este problema pode ser contornado, em parte, fazendo-se orifícios em sua parte inferior, e usando-se uma chama para seu aquecimento. Deste modo, parte dos gases da chama entram no seu interior, aumentando sua temperatura interna ${ }^{93}$. Uma outra variante é a utilização do sistema "flame in tube" $94-95$, onde faz-se queimar, na confluência do tubo $\mathrm{T}$, uma pequena chama $\mathrm{H}_{2} / \mathrm{O}_{2}$, obtida pela introdução lateral destes gases. $\mathrm{O}$ volume de atomização é assim enriquecido em radicais $\mathrm{H}$, facilitando a atomização.

\section{DESEMPENHO}

Baxter e colaboradores ${ }^{50}$ realizaram um estudo do desempenho do acoplamento da cromatografia gasosa com a absorção atômica. Derivaram expressões para descrever as mudanças na 
sensibilidade do detector e na eficiência da separação cromatográfica. Os autores verificaram que os pontos mais importantes para se obter a melhor performance são a escolha da célula de atomização e da coluna cromatográfica. No caso das primeiras, deve-se dar preferência àquelas que utilizem, ao máximo, o volume de atomização definido pelo caminho ótico da fonte primária, o que levará a maiores sensibilidades. Altas sensibilidades podem, no entanto, contribuir para o alargamento do pico, levando a piores eficiências e resoluções. Logo, deve-se encontrar um compromisso entre eficiência cromatográfica e sensibilidade de detecção. Uma maneira de fazer melhorar a resolução é pelo aumento da vazão do gás carreador. Entretanto, o aumento desta vazão pode reduzir a temperatura do atomizador e, assim, reduzir a eficiência de atomização. Quanto à escolha da coluna cromatográfica, deve-se dar preferência a colunas capilares, que irão levar a uma maior resolução e picos mais estreitos e podem ser operadas a menores vazões de gás carreador. Menores vazões podem implicar em aumento da sensibilidade, tanto pelo maior tempo de residência do analito no caminho ótico, como pela menor diminuição da temperatura da célula de atomização, sendo o resultado final a melhoria dos limites de detecção.

\section{AUTOMAÇÃO}

Reações de derivatização relativas às diversas combinações CG-AAS podem ser realizadas em fluxo, desde que a cinética da reação o permita. Quando este é o caso (geração de vários hidretos pela reação com $\mathrm{NaBH}_{4}$, p. ex.), vantagens típicas como maior repetibilidade, menor risco de contaminação, uso de menores quantidades de reagentes e maior rapidez são obtidas ${ }^{96}$. Entretanto, algumas reações de derivatização podem ser excessivamente lentas, dificultando a abordagem em fluxo. Todavia, mesmo os sistemas em batelada podem ser automatizados, o que é desejável, dado o grande número de operações normalmente envolvidas. Assim, Tseng e colaboradores $^{64}$ desenvolveram um processo em batelada, totalmente automatizado, para a especiação de mercúrio em amostras ambientais, combinando derivatização por etilação (ou geração de hidretos), pré-concentração em trap criogênico, separação por desorção térmica em cromatografia gasosa e detecção por absorção atômica em tubo de quartzo (D-CT-GC-QFAAS). A avaliação do sistema automatizado em termos de exatidão e precisão revelou uma melhoria significativa em relação às técnicas não automatizadas. Como resultado, foi possível validar um procedimento geral para a análise de diferentes amostras ambientais (água, sedimento, material em suspensão e tecidos biológicos). Já Donard e colaboradores ${ }^{97}$ desenvolveram um sistema automatizado, também em batelada, para a especiação de Sn inorgânico e compostos alquil-estanho, utilizando geração de hidretos. Este procedimento pode ser aplicado para a especiação de outros elementos formadores de hidretos e também para o mercúrio ${ }^{96,98-99}$.

\section{PREPARO DAS AMOSTRAS}

Embora sistemas CG-AAS apresentem, sistematicamente, bons resultados para soluções analíticas, podem falhar na análise de amostras reais. O sucesso na análise de amostras reais está intimamente ligado a um pré-tratamento adequado, sendo esta etapa, muitas vezes, a determinante da duração, eficiência, precisão e exatidão do procedimento analítico total ${ }^{100}$. Quando a especiação se faz necessária, esta etapa pode ser a principal causa de longos tempos de análise, podendo chegar até dias, ou, por força de perdas ou interconversão das espécies, de baixa precisão ou exatidão.

No caso de amostras líquidas, os principais problemas relacionam-se à sua coleta e conservação. Na determinação de traços totais nestas amostras, a sua acidulação é, em geral, suficiente. Entretanto, no caso da especiação, há o risco de interconversão das espécies. Poucos estudos ${ }^{101-105}$ relatam a melhor forma de conservação destas amostras, sendo necessárias investigações específicas. No caso de amostras sólidas, o maior problema se traduz em definir um procedimento enérgico o suficiente para a extração completa das espécies, sem mudanças de sua forma físico-química. Em alguns casos, estes problemas podem ser aliviados com o uso da automatização. Não está no escopo deste artigo a discussão do pré-tratamento de amostras com vistas à especiação, uma vez que boas revisões podem ser encontradas na literatura ${ }^{106-109}$. Entretanto, deve-se apontar que extrações assistidas por microondas com o uso de soluções ácidas diluídas ${ }^{110-114}$, assim como a microextração em fase sólida (SPME) ${ }^{115-119}$, vem mostrando ser procedimentos bastante promissores.

\section{PRÉ-CONCENTRAÇÃO}

Um outro importante problema, relativo ao acoplamento GC-AAS, pode vir a ser a pequena capacidade das colunas. Neste caso, apenas uma pequena fração da amostra pode ser processada, resultando em perda de sensibilidade do sistema. Se for o caso, esta limitação é contornada pela introdução de uma etapa de pré-concentração, de preferência em linha, previamente à entrada da amostra na coluna cromatográfica. Um exemplo, já discutido, é a utilização de armadilhas criogênicas. Num outro enfoque, Lobinski e Adams ${ }^{73}$ realizaram a pré-concentração utilizando um tubo metálico ("liner") e solvente volátil. A temperatura deste tubo metálico é elevada, a fim de aumentar a pressão de vapor do solvente, e assim mantida por 1-5 minutos, enquanto uma corrente de gás carreador retira o solvente volatilizado, permanecendo os analitos menos voláteis; quando o volume do solvente reduzse a tão somente $1 \mu \mathrm{L}$, ou menos, o tubo metálico é aquecido e os analitos são volatilizados e carreados até a coluna. Se acoplado a uma coluna capilar, este sistema permite uma separação efetiva de picogramas do analito. Em ambos os casos, observa-se a prévia separação analito-matriz, simultaneamente à pré-concentração das várias espécies; a mistura que adentra ao sistema de separação é bem mais simples, minimizando interferências e aumentando a sensibilidade do procedimento analítico total.

Em relação aos procedimentos usuais de pré-concentração, pode-se verificar que apenas alguns trabalhos os associam ao acoplamento CG-AAS, sendo exemplos a utilização de extração por solvente ${ }^{120-121}$ ou extração sólido-líquido ${ }^{120,122}$.

\section{APLICAÇÕES}

A Tabela 1 resume alguns trabalhos envolvendo a utilização de GC acomplada a AAS

\section{CONCLUSÃO}

A importância da especiação química de elementos traços é hoje plenamente reconhecida, sendo que o seu desenvolvimento metodológico, iniciado mais sistematicamente nos anos 70 , apresentou, na última década, um progresso bastante significativo. Entre os diversos enfoques adotados, o acoplamento CGAAS, quando aplicável, apresenta-se como o mais adequado a análises de rotina, considerando-se custo e tempo além, naturalmente, dos requisitos de sensibilidade, precisão, exatidão e respostas espécie-específicas. Em relação à detecção, o uso de tubos de quartzo aquecidos é o que reúne o maior conjunto de vantagens, permitindo a especiação dos elementos mais importantes em estudos atuais ( $\mathrm{Pb}, \mathrm{Hg}$, As, Se etc). Apesar do grande potencial instrumental disponível, problemas relativos ao pré-tratamento de amostras reais persistem, e devem ser sempre devidamente considerados para uma análise bem sucedida. 
Tabela 1.

\begin{tabular}{|c|c|c|c|c|c|}
\hline ESPÉCIES & MATRIZ & TÉCNICA & COLUNAS & Obs & REF \\
\hline $\begin{array}{l}\text { Sb } \\
\mathrm{Sb}(\mathrm{III}), \mathrm{Sb}(\mathrm{V}) \text {, ácido } \\
\text { metil-estibônico e } \\
\text { ácido dimetilestibínico }\end{array}$ & $\begin{array}{l}\text { Águas } \\
\text { naturais }\end{array}$ & HG-CG-GFAAS & $\begin{array}{l}\text { Tubo em } U \text { de } \\
\text { pirex, resfriado em } \\
\mathrm{N}_{2} \text { líquido }\end{array}$ & $\begin{array}{l}\text { Diferenciação do } \\
\mathrm{Sb}(\mathrm{III}) \text { e } \mathrm{Sb}(\mathrm{V}) \\
\text { utilizando controle } \\
\text { de pH }\end{array}$ & 123 \\
\hline Metil-Sb(V) & Padrões & CT-CG-AAS & & & 124 \\
\hline $\begin{array}{l}\text { Se } \\
\text { Dimetil-Se } \\
\text { Dimetil-di-Se }\end{array}$ & $\mathrm{Ar}$ & GC-GFAAS & $\begin{array}{l}10 \% \text { polimetafenileter } \\
\text { em Chromosorb W }\end{array}$ & & 125 \\
\hline Organo-Selênicos & Padrões & $\begin{array}{l}\text { Etilação-CT- } \\
\text { GC-GFAAS }\end{array}$ & & & 126 \\
\hline $\begin{array}{l}\text { Dimetil-Se } \\
\text { Dietil-Se } \\
\text { Dimetil-di-Se }\end{array}$ & Solo & GC-GFAAS & $\begin{array}{l}1.5 \% \text { OV-1 em } \\
\text { Shimalite W }\end{array}$ & $\begin{array}{l}\text { Forno revestido } \\
\mathrm{Pd} / \mathrm{Zr}\end{array}$ & 127 \\
\hline $\begin{array}{l}\text { As } \\
\text { Organo-Arsênicos }\end{array}$ & Padrões & Hg-CT-QFAAS & $\begin{array}{l}\text { OV-3 em } \\
\text { Chromosorb W-HP }\end{array}$ & & 128 \\
\hline $\begin{array}{l}\text { Hg } \\
\text { Metil-Hg } \\
\text { Hg inorgânico }\end{array}$ & $\begin{array}{l}\text { Sedimentos } \\
\text { Águas } \\
\text { Tecidos biológicos }\end{array}$ & $\begin{array}{l}\text { HG-CT-GC- } \\
\text { QFAAS }\end{array}$ & $\begin{array}{l}\text { Trap tubo em } \mathrm{U} \\
\text { vidro resfriado em } \\
\mathrm{N}_{2} \text { líquido }\end{array}$ & $\begin{array}{l}\text { Procedimento } \\
\text { on-line } \\
\text { automatizado }\end{array}$ & 64 \\
\hline Metil-Hg & $\begin{array}{l}\text { Sedimentos } \\
\text { Amostras } \\
\text { biológicas }\end{array}$ & HG-GC-AAS & $\begin{array}{l}\text { Capilar revestida } \\
\text { com, CP-SIL 5CB }\end{array}$ & $\begin{array}{l}\text { Extração fase sólida, } \\
\mathrm{HG} \text { com } \mathrm{KBH}_{4}\end{array}$ & 68 \\
\hline Organo mercuriais & $\begin{array}{l}\text { Amostras } \\
\text { ambientais }\end{array}$ & $\begin{array}{l}\text { D-CT-GC- } \\
\text { QFAAS }\end{array}$ & $\begin{array}{l}10 \% \text { SP- } 2100 \mathrm{em} \\
\text { Chromosorb } \\
\text { G AW-DMCS }\end{array}$ & Sistema automatizado & $\begin{array}{l}98 \\
99\end{array}$ \\
\hline $\mathrm{Hg}$ & Sedimentos & $\begin{array}{l}\text { Etilação-CT-GC- } \\
\text { QTAAS }\end{array}$ & $\begin{array}{l}10 \% \text { SP-2100 em } \\
\text { Chromosorb W-HP }\end{array}$ & & 129 \\
\hline $\mathrm{Hg}$ & Padrões & $\begin{array}{l}\text { Etilação-CT-GC- } \\
\text { QTAAS }\end{array}$ & $\begin{array}{l}\text { Megabore revestida } \\
\text { com DB- } 624\end{array}$ & & $\begin{array}{l}17 \\
130\end{array}$ \\
\hline $\begin{array}{l}\text { Metil-Hg } \\
\text { Hg inorgânico }\end{array}$ & $\begin{array}{l}\text { Amostras } \\
\text { aquosas }\end{array}$ & GC-QTAAS & & Etilação & 7 \\
\hline $\begin{array}{l}\mathrm{CH}_{3} \mathrm{HgCl} \\
\mathrm{C}_{2} \mathrm{H}_{5} \mathrm{HgCl} \\
\mathrm{Hg} \text { inorgânico }\end{array}$ & Padrões & GC -GFAAS & $\begin{array}{l}\text { WCOT revestida com } \\
\text { OV-1 }\end{array}$ & $\begin{array}{l}\text { Pre-concentração em } \\
\text { ditizona ligada a } \\
\text { resina acrílica }\end{array}$ & 69 \\
\hline $\begin{array}{l}\text { Sn } \\
\text { Organo estânicos }\end{array}$ & Sedimentos & HG-CT-GC-AAS & $\begin{array}{l}10 \% \text { Supelco SP2100 } \\
\text { em Chromosorb W HP }\end{array}$ & & 65 \\
\hline Butil-Sn & Sedimentos & D-CT-GF-QTAAS & $\begin{array}{l}\text { Trap tubo em } \mathrm{U} \text { pirex } \\
\text { resfriado em } \mathrm{N}_{2} \text { líquido }\end{array}$ & Extração metanol & 59 \\
\hline Butil-Sn & Sedimentos & $\begin{array}{l}\text { Etilação-GC } \\
\text { capilar-QFAAS }\end{array}$ & HP-1 & $\begin{array}{l}\text { Abertura com } \\
\text { microondas e } \\
\text { extração com hexano }\end{array}$ & 100 \\
\hline
\end{tabular}


cont. Tabela 1.

\begin{tabular}{|c|c|c|c|c|c|}
\hline ESPÉCIES & MATRIZ & TÉCNICA & COLUNAS & Obs & REF \\
\hline Organo estânicos & Sedimentos & $\begin{array}{l}\text { Etilação-GC- } \\
\text { QFAAS }\end{array}$ & Capilar & $\begin{array}{l}\text { Complexação com } \\
\text { tropolona seguida } \\
\text { de extração }\end{array}$ & 70 \\
\hline $\begin{array}{l}\text { Dibutil-Sn } \\
\text { Tributil-Sn }\end{array}$ & Sedimentos & $\begin{array}{l}\text { D-CT-GC- } \\
\text { QTAAS }\end{array}$ & $\begin{array}{l}3 \% \text { SP2 } 100 \text { em } \\
\text { Chromosorb G } \\
\text { AW DMCS }\end{array}$ & Etilação & 58 \\
\hline $\begin{array}{l}\text { Monobutil-Sn } \\
\text { Dibutil-Sn } \\
\text { Tributil-Sn }\end{array}$ & $\begin{array}{l}\text { Lodo } \\
\text { Esgoto }\end{array}$ & D-GC-GFAAS & $\begin{array}{l}\text { Capilar revestida } \\
\text { com dimetil- } \\
\text { poli-siloxano }\end{array}$ & $\begin{array}{l}\text { Extração: tropolone } \\
\text { em tolueno } \\
\text { Etilação }\end{array}$ & 71 \\
\hline Butil-Sn & Conchas & HG-CT-QTAAS & $\begin{array}{l}10 \% \text { OV-1 em } \\
\text { Chromosorb W HP }\end{array}$ & Sonicação & 66 \\
\hline Organo estânicos & $\begin{array}{l}\text { Óleos } \\
\text { comestíveis }\end{array}$ & GC-QT-AAS & $\begin{array}{l}3 \% \text { OV-7 em } \\
\text { Chromosorb W HP }\end{array}$ & $\begin{array}{l}\text { Extração: tropolona/ } \\
\text { metanol (reação } \\
\text { Grignard) 3\%OV-7 } \\
\text { em Chromosorb W }\end{array}$ & 131 \\
\hline $\begin{array}{l}\text { Metil-Butil Sn } \\
\text { n-butil Sn }\end{array}$ & Águas & GC-AAS & $\begin{array}{l}3 \% \text { OV-1 em } \\
\text { Chromosorb W }\end{array}$ & $\begin{array}{l}\text { Derivatização com } \\
\text { reagentes de Grignard } \\
\text { Flame-in-tube }\end{array}$ & 53 \\
\hline Organo estânicos & Águas & HG-CT-GC-AAS & $\begin{array}{l}3 \% \text { SP } 2100 \text { em } \\
\text { Chromosorb G } \\
\text { AW-DMCS }\end{array}$ & Sistema automatizado & 97 \\
\hline Organo estânicos & Padrões & HG-GC-AAS & $\begin{array}{l}\text { Capilar revestida } \\
\text { com metil-silicone }\end{array}$ & Flame-in-tube & 72 \\
\hline Organo estânicos & Padrões & HG-GC-QFAAS & $\begin{array}{l}\text { Trap tubo em } \mathrm{U} \\
\text { resfriado em } \mathrm{N}_{2} \text { líquido }\end{array}$ & & 132 \\
\hline $\mathbf{P b}$ & & & & & \\
\hline $\begin{array}{l}\mathrm{Pb} \text { alquila } \\
\mathrm{Pb} \text { tetra alquila }\end{array}$ & $\begin{array}{l}\text { Águas } \\
\text { Mexilhão }\end{array}$ & GC-QTAAS & $\begin{array}{l}10 \% \text { OV-101 em } \\
\text { Chromosorb W }\end{array}$ & $\begin{array}{l}\text { Extração } \\
\text { Propilação }\end{array}$ & 78 \\
\hline Organo plúmbicos & Padrões & HG-GC -AAS & $\begin{array}{l}\text { Capilar revestida } \\
\text { com metilsilicone }\end{array}$ & Flame-in-tube & 72 \\
\hline $\begin{array}{l}\text { Dietil- } \mathrm{Pb} \\
\text { Trietil- } \mathrm{Pb} \\
\mathrm{Pb} \text { inorgânico }\end{array}$ & Urina & $\begin{array}{l}\text { HG-CT-GC- } \\
\text { QTAAS }\end{array}$ & & & 133 \\
\hline Alquil- $\mathrm{Pb}$ & Águas & GC-QTAAS & $\begin{array}{l}\text { OV-101 em } \\
\text { Chromosorb W }\end{array}$ & $\begin{array}{l}\text { Derivatização com } \\
\text { reagentes de Grignard }\end{array}$ & 56 \\
\hline $\mathrm{Pb}$ & Águas & $\begin{array}{l}\text { Etilação-CT-GC } \\
\text {-QTAAS }\end{array}$ & $\begin{array}{l}\text { Trap tubo em U } \\
\text { resfriado em } \\
\mathrm{N}_{2} \text { líquido }\end{array}$ & & $\begin{array}{l}57 \\
134\end{array}$ \\
\hline
\end{tabular}

\section{GLOSSÁRIO}

AAS Espectrometria de Absorção Atômica

AES Espectrometria de Emissão Atômica

AFS Espectrometria de Fluorescência Atômica

CG Cromatografia Gasosa

CT Trap criogênico

D Derivatização

GFAAS Espectrometria de Absorção Atômica com forno de grafite
HG Geração de hidretos

HPLC Cromatografia líquida de alta resolução

ICP-AES Espectrometria de emissão atômica com fonte de plasma induzido

ICP-MS Espectrometria de massa com fonte de plasma induzido

QTAAS Espectrometria de Absorção Atômica com tubo de quartzo

MIP Plasma induzido por microondas

SPME Micro-extração em fase sólida 


\section{REFERÊNCIAS}

1. Ure, A. M.; Heavy metals in soil, Blackie, Alloway Bj, 1990, 71.

2. Moore, J. W.; Ramamoorthy, S.; Heavy metals in naturals waters, applied monitoring and impact assessment, eds. Moore, J.W., and Ramamoorthy, S., Springer-Verlag, New York, 1984, pp. 125.

3. Hempel, M.; Chau, Y. K.; Dutka, B. J.; Mc Innis, R.; Lui, D.; Kwan, K. K.; Analyst 1995, 120, 721.

4. Harrison, R. M.; Rapsomanikis, S.; Environmental analysis using chromatography interfaced with atomic spectroscopy, ed. Harrison R. M. and Rapsomanikis S., Ellis Horwood, Chichester, 1989, 189.

5. Chiavarini, S.; Cremisini, C.; Morabito, R.; Element speciation in bioinorganic chemistry, ed. by Sergio Caroli, Wiley-Interscience publication, 1996, 289.

6. Caroli, S.; La Torre, F.; Petrucci, F.; Violante, N.; Element speciation in bioinorganic chemistry, ed. by Sergio Caroli, Wiley-Interscience publication, 1996, 445.

7. Rapsomanikis, S.; Craig, P. J.; Anal. Chim. Acta 1991, $248,563$.

8. Harrison, R. M; Rapsomanikis, S.; Environmental analysis using chromatography interfaced with atomic spectrometry, ed. Harrison R.M. and Rapsomanikis S., Ellis Horwood, Chichester, 1989, 299.

9. Baxter, D. C.; Frech, W; Analyst 1993, 118, 495.

10. Kato, T.; Uehiro, T.; Yashura, A; Morita, M.; J. Anal. At. Spectrom. 1992, 7, 15.

11. Tao, H.; Murakami, T.; Tominaga, M.; Miyazaki, A; J. Anal. At. Spectrom. 1998, 13, 1085.

12. Amouroux, D.; Tessier, E.; Pecheyran, C.; Donard, O. F. X.; Anal. Chim. Acta 1998, 377, 241.

13. Heisterkamp, M.; De Smaele, T.; Candelone, J. P.; Moens, L.; Dams, R.; Adams, F. C.; J. Anal. At. Spectrom. 1997, 12,1077

14. Feldmann, J. ; J. Anal. At. Spectrom. 1997, 12, 1069.

15. Moens, L.; De Smaele, T.; Damsm R.; Van den Broeck, P.; Sandra, P.; Anal. Chem. 1997, 69, 1604.

16. Gallus, S. M.; Heumann, K. G.; J. Anal. At. Spectrom. 1996, 11,887 .

17. Hintelmann, H. E.; Evans, D.; Villeneuve, R. D.; J. Anal. At. Spectrom. 1995,10, 619.

18. Prange, A; Jantzen, E.; J. Anal. At. Spectrom. 1995 10, 105.

19. De Smaele, T.; Verrept, P.; Dams, R.; Spectrochim. Acta, Part B 1995, 50, 1409.

20. Kim, A; Foulkes, W.; Ebdon, M. E.; J. Anal. At. Spectrom. 1992, 7, 1147.

21. Kim, A.; Hill, L.; J. High Resolut. Chromatogr. 1992, 10,665 .

22. Jerrell, L. J.; Dunn, M. R.; Fannin, H. B.; Appl. Spectrosc. 1999, 53, 245

23. Junyapoon, S.; Ross, A. B.; Cooke, M.; J. High Resolut. Chromatogr. 1999, 22, 47.

24. Pereiro, I. R.; Wasik, A.; Lobinski, R.; J. Anal. At. Spectrom. 1998, 13, 743.

25. Orellana-Velado, N. G.; Pereiro, R.; Sanz-Medel, A; J. Anal. At. Spectrom. 1998, 13, 905.

26. Jimenez, M. S.; Sturgeon, R. E.; J. Anal. At. Spectrom. 1997, 12, 597.

27. Ceulemans, M.; Adams, F. C.; J. Anal. At. Spectrom. 1996, 11, 201

28. Ceulemans, M.; Lobinski, R.; Dirkx, W. M. R.; Fresenius J. Anal. Chem. 1993, 347, 256.

29. Lobinski, R.; Bourtron, C. F.; Candelone, J. P.; Hong, S.; Lobinska J. S.; Adams, F. C.; Anal. Chem. 1993, 65, 2510.

30. Cai, Y.; Jaffé, R.; Alli, A; Jones, R. D.; Anal. Chim. Acta 1996, 334, 251 .
31. Emterborg, H; Bjorklund, E.; Odman, F.; Karlsson, L.; Mathiasson, L.; Frech, W.; Baxter, D. C.; Analyst 1996, 121, 19.

32. Pereiro, I. R.; Wasik, A.; Lobinski, R.; J. Chromatogr. 1998, 795, 359.

33. Szpunar, J.; Schmitt, V.; Monod, J. L.; J. Anal. At. Spectrom. 1996, 11, 193.

34. Minganti, V.; Capelli, R.; De Pellegrini, R.; Fresenius J. Anal. Chem 1995, 351, 471.

35. Mena, M. L.; McLeod, C. W.; Quevauviller, P.; Fresenius' J. Anal. Chem 1995, 351, 456.

36. de la Calle-Guntinas, M. B.; Lobinski, R.; Adams, F. C.; J. Anal. At. Spectrom. 1995, 10, 111.

37. Tutschku, S.; Mothes, S.; Dittrich, K.; J. Chromatogr. 1994, 683, 269.

38. Szpunar, J.; Ceulemans, M.; Lobinski, R.; Adams. F. C.; Anal. Chim. Acta 1993, 278, 99.

39. Lobinski, R.; Adams, F. C; Anal. Chim. Acta 1992, 262, 285.

40. Heisterkamp, M.; Adams, F. C.; J. Anal. At. Spectrom. 1999, 14, 1307.

41. Heisterkamp, M.; Adams, F. C.; Fresenius J. Anal. Chem. 1998, 362, 489.

42. Mothes, S.; Wennrich, R.; J. High Resolut. Chromatogr. 1999, 22, n 3181.

43. Frech, W.; Snell, J. P.; Sturgeon, R. E.; J. Anal. At. Spectrom. 1998, 13, 1347.

44. Pereiro, I. R.; Lobinski, R. ; J. Anal. At. Spectrom. 1997, 12, 1381.

45. De La Calle-Guntinas, M. B.; Brunori, C.; Scerbo, R.; Chiavarini, S.; Quevauviller, P.; Adams, F.; Morabito, R.; J. Anal. At. Spectrom. 1997, 12,1041.

46. Snell, J. P.; Frech, W.; Thomassen, Y.; The Analyst, 1996, 121,1055

47. Welz, B.; J. Anal. At. Spectrom. 1998, 13, 413.

48. Tsalev, D. L.; J. Anal. At. Spectrom. 1999, 14, 147.

49. Emterborg, H.; Sinemus, H.; Radzuik, B.; Baxter, D. C.; Frech, W.; Spectrochim. Acta, Part B 1996, 51, 829.

50. Baxter, D. C.; Wolfgang, F.; Analyst 1993, 118, 495

51. Guardia, M. D. L.; Element Speciation in Bioinorganic Chemistry, ed. by Sergio Caroli, Wiley-Interscience publication, 1996, 105

52. Caroli, S.; La Torre, F.; Petrucci, F.; Violante, N.; Element speciation in bioinorganic chemistry, ed. by Sergio Caroli, Wiley-Interscience publication, 1996, 453.

53. Chau, Y. K.; Wong, P. T. S.; Bengert, G. A; Anal. Chem. 1982, 54, 246 .

54. Dirkx, W. M. R.; Lobinski, R.; Adams, F. C.; Anal. Sci. 1993, 9, 12.

55. Dirkx, W. M. R.; Adams, F. C.; Mikrochim. Acta 1992 , 109, 79.

56. Chakraborti, D.; De Jonghe, W.; Van Mol W. E.; Cleuvenbergen R. J. A. V.; Adams, F. C.; Anal. Chem. 1984, 56, 2692.

57. Rapsomanikis, S.; Donard, O. X. F.; Weber, J. H.; Anal. Chem. 1986, 58, 35.

58. Cai, Y.; Rapsomanikis, S.; Andreae, M. O.; J. Anal. At. Spectrom. 1993, 8, 119.

59. Cai, Y.; Rapsomanikis, S.; Andreae, M. O.; Anal. Chim. Acta 1993, 274, 243.

60. Clark, S.; Craig, P. J.; Mikrochim. Acta 1992, 109, 141.

61. Ariza, J. L. G.; Morales, E.; Sanchez-Rodas D.; Gerladez I.; Trends Anal. Chem. 2000, 19, 200.

62. Pons, B., Carrera, A ; Nerin C.; J. Chromatogr. B 1998, $1-2,139$.

63. Agterdenbos, J.; Bax, D.; Anal. Chim. Acta 1986, 188, 127. 64. Tseng, C. M.; Diego, A; Pinaly, H.; Amouroux, D.; Donard O. F. X.; J. Anal. At. Spectrom. 1998, 13, 755.

65. Martin, F. M.; Tseng, C.; Belin, C.; Quevauviller, P.; Donard, O. F. X.; Anal. Chim. Acta 1994, $286,343$. 
66. Pannier, F.; Astruc, A.; Astruc, M.; Anal. Chim. Acta 1994, 287, 17.

67. Vien, S. H.; Frey R. C.; Anal. Chem. 1988, 60, 465

68. Bin, H.; Gui-bin, J.; Zhe-ming, N.; J. Anal. At. Spectrom. 1998, 13, 1141.

69. Salih, B.; Say, R.; Denizli, A; Genc, O ; Piskin, E.; Anal. Chim. Acta. 1998, 371, 177.

70. Ceulemans, M.; Slaets, S.; Adams, F.; Talanta 1998, 46, 395.

71. Chau, Y. K.; Zhang, S.; Maguire, R. J.; The Analyst 1992, 117, 1161.

72. Foster, R. C.; Howard, A. G.; Analytical Proceedings 1989, 26, 34.

73. Lobinski, R.; Adams, F. C.; J. Anal. At. Spectrom. 1992, 7, 987.

74. Desauziers, V.; Tese, Pau University, 1991.

75. Hewitt, C. N.; Harrison, R. M.; Anal. Chim. Acta 1985, 167, 277.

76. Radziuk, B; Thomassen, Y.; Van Loon, J. C.; Chau, Y. K.; Anal. Chim. Acta 1979, 105, 255.

77. Robinson, J. W.; Kiesel, E. L.; Goodbread, J. P.; Bliss R.; Marshall R.; Anal. Chim. Acta 1977, 92, 321.

78. Mikac, N.; Wang, Y.; Harrison, R. M.; Anal. Chim. Acta 1996, 326, 57.

79. Segar, D. A; Anal. Lett. 1974, 7, 89.

80. Rapsomanikis, S.; Environmental Analysis using chromatography interfaced with atomic spectrometry, ed. Harrison, R. M. and Rapsomanikis S., Ellis Horwood, Chichester, 1989, 55.

81. Emterborg, H.; Sinemus, H. W.; Radzuik, B.; Baxter, D. C.; Frech, W.; Spectrochim. Acta, Part B 1996, 51, 829.

82. Kolb, B.; Kemmner, G.; Schleser, F. H.; Wiedeking, E.; Z. Anal. Chem. 1966, 21, 166.

83. Gonzalez, J. G.; Ross, R. T.; Anal. Lett. 1972, 5, 683.

84. Longbottom, J. E.; Anal. Chem. 1972, 44, 111.

85. Katou, T.; Nakagawa, R.; Bull. Inst. Environ. Sci. Technol. 1974, 1, 19.

86. Chau, Y. K.; Wong, P. T. S.; Saitoh, J.; J. Chromatog. Sci 1976, 14, 162 .

87. Coker, D. T.; Anal. Chem. 1975, 47, 386.

88. Robinson, J. W.; Vidaurreta, L. E.; Wolcott, D. K.; Kiesel, E.; Spectrosc. Lett. 1975, 8, 491.

89. Bye, R.; Paus, P. E.; Solberg, R.; Thomassen, Y.; At. Abs. Newsl. 1978, 17, 131.

90. De Jonghe, W. R. A; Chakraborti, D.; Adams F.; Anal. Chim. Acta 1980, 115, 89.

91. Chau, Y. K.; Wong, P. T. S.; Goulden, P. D.; Anal. Chim. Acta 1976, 85, 421.

92. Forsyth, D. S.; Marshall, W. D.; Anal. Chem. 1985, 57, 1299.

93. Grinberg, P.; Campos, R. C.; J. Anal. At. Spectrom. 1999, 14,827

94. Siemer, D. D.; Hageman L.; Anal. Lett. 1975, 8, 323.

95. Alvarado, J.; Jaffe, R.; J. Anal. At. Spectrom. 1998, 13, 1297.

96. Campanella, L.; Pyrzynska ,K.; Trojanowicz, M.; Talanta 1996, 43, 825 .

97. Donard, O. X. F; Rapsomanikis, S.; Weber, J. H.; Anal. Chem. 1986, 58, 772 .

98. Puk, R.; Weber, J. H.; Anal. Chim. Acta 1994, 292, 175.

99. Tseng; C. M.; De Diego, A; Martin, F. M.;Amouroux D.; Donard, O. F. X.; J. Anal. At. Spectrom. 1997, 12, 743.

100. Szpunar, J.; Ceulemans, M.; Schmit, V. O.; Adams, F. C.; Lobinski, R.; Anal. Chim. Acta 1996, 332, 225.

101. Heninger, I.; Potin-Gautier, M.; Gregori, I.; Pinochet, H; Fresenius J. Anal. Chem. 1997, 357, 600.
102. Lanses, P.; Meuleman, C.; Baeyens, W.; Anal. Chim. Acta 1990, 229, 281.

103. Ahmed, R.; Stoeppler, M.; Anal. Chim. Acta 1987, 192, 109.

104. Leermakers, M.; Lansens, P.; Baeyens, W.; Fresenius J. Anal. Chem 1990, 336, 655.

105. Ariza, J. L. G.; Morales, E.; Sanchez-Rodas, D.; Geraldez, I.; Trends Anal. Chem. 2000, 19, 200.

106. Bock, R.; A Handbook of decomposition methods in Analytical Chemistry, Glasgow, International Textbook, 1979.

107. Anderson R.; Sample pretreatment and separation, Analytical Chemistry by open learning, Chichester, Jonh Wiley, 1991.

108. Iyengar, G. V.; Sansoni, B.; Sample preparation of biological material for trace element analysis, Vienna, International Atomic Energy Agency, 1980.

109. Cornelis, R.; Kimpe, J.; Zhang, X.; Spectrochim. Acta, Part B 1999, 53, 187.

110. Lorenzo, R. A.; Vazquez, M. J.; Cela, R.; Trends Anal. Chem. 1999, 18, 410.

111. Ackley, K. L.; B'Hymer, C.; Sutton K. L.; Caruso, J. A.; J. Anal. At. Spectrom. 1999, 14, 845.

112. Martinez-Garcia, M. L.; Zubieta, A. C.; Rodriguez, D. P.; Anal. Let. 1999, 32, 139.

113. Guo, T.; Baasner J.; Anal. Chim. Acta 1993, 278, 189.

114. Koch H. F.; Girard, L. A; Roundhill, D. M.; At. Spectrom. 1999, 20, 33.

115. He, B; Jiang, G. B.; Fresenius J. Anal. Chem. 1999, 365, 615.

116. Fragueiro, M. S.; Alava-Moreno, F.; Lavilla, I.; Bendicho, C.; J. Anal. At. Spectrom. 2000, 15, 705.

117. Bin, H.; Gui-bin, J.; Zhe-ming, N.; J. Anal. At. Spectrom. 1998, 13, 1141.

118. Snell, J. P.; Frech, W.; Yngvar, T.; Analyst 1996, 121, 1055.

119. Moens, L.; De Smaele, T.; Dams, R.; Broeck, P. V. D.; Sandra, P; Anal. Chem. 1997, 69, 1604.

120. Chakraborti, D.; De Jonghe, W. R. A; Van Mol, W. E.; Van Cleuvenbergen, R. J. A; Adams, F. C; Anal. Chem. 1984, 56, 2692.

121. Van Cleuvenbergen, R. J. A.; Chalraborti, D.; Adams, F. C.; Anal. Chim. Acta 1990, 228, 77.

122. Chau, Y. K.; Wong, P. T. S.; Bengert, G. A ; Dunn, J. L.; Anal. Chem. 1984, 56, 271.

123. Andreae, M. O; Foster, P.; Asmodé, J. F.; Anal. Chem. 1981, 53, 1766.

124. Dodd, M.; Grundy, S. L.; Reimer, K. J.; Cullen, W. R.; Appl. Organomet. Chem. 1992, 6, 207.

125. Jiang, S.; De Jonghe, W.; Adams, F.; Anal. Chim. Acta 1982, 136, 183.

126. Alabash, I. R.; Rendl, J.; Grasserbauer, M.; Fresenius J. Anal. Chem. 1998, 360, 723.

127. Gui-bin, J.; Zhe-ming, N..; Zhang, L.; Ang, Li; Heng-bin H.; Xiao-quan, S.; J. Anal. At. Spectrom. 1992, 7, 447.

128. Howard, A. G.; Salou, C.; J. Anal. At. Spectrom. 1998, 13,683 .

129. Tseng, C. M.; De Diego, A; Martin, F. M;. Donard, O F. X.; J. Anal. At. Spectrom. 1997, 12, 629.

130. Fischer, R.; Rapsomanikis, S.; Andreae M. O; Anal. Chem. 1993, 65, 763 .

131. Forsyth, D. S.; Weber, D.; Dalglish, K; Talanta 1993, 40, 299.

132. Sarradin, P. M.; Leguille, F.; Astruc, A; Pinel, R.; Astruc, M.; Analyst 1995, 120, 79.

133. Yamaguchi, H.; Arai, F.; Yamumara, Y.; Ind. Health 1981, 19, 115.

134. Donard, O F. X.; Randall, L.; Rapsomanikis, S.; Int. J. Environ. Anal. Chem. 1986, 27, 55. 